\title{
Metahemoglobinemia durante a utilização de óxido nítrico em criança submetida a transplante cardíaco: relato de caso
}

\author{
Acquired methemoglobinemia during nitric oxide utilization in a pediatric \\ heart transplantation: case report
}

\author{
Lívia Rossetti de Abreu e Limaํㅜ, Filomena Regina Barbosa Gomes Gallas²
}

\begin{abstract}
Abreu e Lima LR, Gallas FRBG. Metahemoglobinemia durante a utilização de óxido nítrico em criança submetida a transplante cardíaco: relato de caso / Acquired methemoglobinemia during nitric oxide utilization in a pediatric heart transplantation: case report. Rev Med (São Paulo). 2017 abr.-jun.;96(2):125-30.
\end{abstract}

RESUMO: Paciente de 7 anos, sexo masculino, portador de miocardiopatia restritiva relacionada ao gene BAG3 e histórico de hipertensão pulmonar com insuficiência tricúspede importante, admitido para avalição de transplante cardíaco. Após a cirurgia deu entrada na UTI estável mas evoluiu no $1^{\circ} \mathrm{PO}$ com acidose metabólica, choque refratário ao volume e hipoxemia refratária a manobras de fisioterapia. Os níveis de metahemoglobina foram dosados e o óxido nítrico foi imediatamente suspenso. Iniciou-se administração endovenosa de azul de metileno na dose $3 \mathrm{mg} / \mathrm{kg}$ com melhora progressiva do quadro clínico. Os níveis de metahemoglobina eram 29,05 e 5,2 ao início e final da administração da droga, respectivamente. Conclusão: Metahemoglobinemia deve ser suspeitada em pacientes que utilizam NO. Níveis elevados de metahemoglobina no sangue podem ser incompatíveis com a vida. O tratamento deve ser instituído de forma rápida e eficaz.

Descritores: Óxido nítrico; Metemoglobina/análise; Transplante de coração; Hemoglobinas; Metemoglobinemia; Criança.

\begin{abstract}
A 7-year-old boy was admitted for evaluation to heart transplantation with a restrictive cardiopathy congenital associated to BAG3 gene. During childhood, he developed high pulmonary hipertension with a significant tricuspid insufficiency. At first post-operative, patient has evoluted with metabolic acidosis, shock refractory to volume and refractory hypoxemia presented at maneuvers physiotherapy. The levels of methemoglobin were checked on laboratory and the nitric oxide was immediately suspended. It started the administration of methylene blue in $3 \mathrm{mg} / \mathrm{kg}$ with progressive improvement of clinical situation. Levels of metheboglobin were 29,05 before the treatment and 5,2 after methylene blue administration. Conclusion: Metahemoglobinemia must be suspected in patients using NO. Elevated levels of metahemoglobin may be incompatible with life. Treatment should be instituted quickly and effectively.
\end{abstract}

Keywords: Nitric oxide; Methemoglobin/analysis; Heart transplantation; Hemoglobins; Methemoglobinemia; Child.

O trabalho é vinculado à Liga de Anestesiologia, Dor e Terapia Intensiva. Trabalho apresentado em formato de comunicação oral no 63 Congresso Brasileiro de Anestesiologia, Brasília, DF, 10-14 nov. 2016.

1. Estudante de Medicina da Faculdade de Medicina da Universidade de São Paulo - FMUSP. Membro da Liga de Anestesiologia, Dor e Terapia Intensiva. E-mail: livia.rossetti@fm.usp.br.

2. Livre-Docente em Medicina na área de Anestesiologia pela Faculdade de Medicina da Universidade de São Paulo, Supervisora da Unidade de Terapia Intensiva Cirúrgica e do Serviço de Anestesiologia do INCOR - Instituto do Coração do Hospital das Clínicas da Faculdade de Medicina da Universidade de São Paulo. Coordenadora da UTI Cardiológica e Anestesiologista do Hospital Sírio Libanês, Coordenadora da UTI Geral do Instituto do Câncer (ICESP) da Faculdade de Medicina da Universidade de São Paulo. E-mail: filomenagalas@hotmail.com.

Endereço para correspondência: Dra. Filomena Gallas. Instituto do Coração do Hospital das Clínicas, Faculdade de Medicina da Universidade de São Paulo. Av. Dr. Enéas de Carvalho Aguiar, 44 - Cerqueira Cesar. CEP: 05403-000 - São Paulo, SP, Brasil. 


\section{INTRODUÇÃO}

A utilização clínica de óxido nítrico (NO) é consagrada nos casos em que se busca rápida indução de vasodilatação, redução da pressão da artéria pulmonar e aumento dos níveis de oxigenação sanguínea. Seu potencial terapêutico se deve principalmente à alta taxa de solubilidade e ampla capacidade de distribuição e de ação parácrina. Dentre as complicações destacam-se a redução da produção de surfactantes, hipertensão pulmonar de rebote e metahemoglobinemia. Níveis de metahemoglobina (MetHb) acima dos 2\% são potencialmente fatais, especialmente em crianças. Condições genéticas podem levar a metahemoglobinemia congênita e desequilíbrios entre fatores de oxidantes externos e redução do poder redutor endógeno são causadores da forma adquirida da patologia. Nesses casos recomenda-se o reconhecimento precoce do quadro, suspensão imediata do fator desencadeante e administração (nem sempre eficaz) de azul de metileno, na dose adequada.

\section{RELATO DE CASO}

M.F. G. 7 anos e 7 meses de idade com diagnóstico cardiomiopatia restritiva (miopatia miofribrilar relacionada ao gene BAG3) e insuficiência tricúspide importante, encaminhado ao Instituto do Coração (InCor) do Hospital das Clínicas da Faculdade de Medicina da Universidade de São Paulo (HCFMUSP) para transplante cardíaco. A cirurgia foi realizada em 05/05/2015 sem intercorrências, foi feita anestesia balanceada, o tempo de circulação extracorpórea foi de 106 minutos; de anóxia 82 minutos; de isquemia do órgão de 107 minutos. Utilizado cetamina, fentanil, pancurônio, midazolan e sevoflurano em doses habituais. Foi utilizado óxido nítrico na retirada da circulação extracorpórea para controle da hipertensão pulmonar. A criança deu entrada na UTI estável, sedada e sob ventilação mecânica, recebendo dobutamina, adrenalina e óxido nítrico. Evolui no $1^{\mathrm{o}}$ pós-operatório (PO) com, acidose metabólica com necessidade de adequação da volemia e de doses crescentes de fármacos vasoativos e da associação de vasopressina, por choque refratário. No $2^{\circ} \mathrm{PO}$ apresentou diminuição da saturação arterial de oxigênio $\left(\mathrm{SaO}_{2}=99 \%\right),(77,4 \%, 80,5 \%, 81 \%)$, sendo aumentado a fração inspirada de oxigênio $\left(\mathrm{FIO}_{2}\right)$, sem melhora da saturação periférica de oxigênio $\left(\mathrm{SPO}_{2}\right)$. Colhido gasometria que evidenciou pressão parcial de oxigênio no sangue arterial $\left(\mathrm{PAO}_{2}\right)$ de $180 \mathrm{mmHg}$, Saturação periférica de $\mathrm{O}$ de $80 \%$, mostrando discrepância entre $\mathrm{PAO}_{2} / \mathrm{SPO}_{2}$. Sendo feita a hipótse diagnóstica de metahemoglobinemia, optado por administrar via endovenosa azul de metileno na dose de $3 \mathrm{mg} / \mathrm{kg}$ em 30 minutos. A coleta sanguínea de metahemoglobina foi realizada na sequência e encaminhado para o centro de toxicologia para confirmação diagnóstica. Houve recuperação imediata da $\mathrm{SPO}_{2}$. da perfusão periférica e do livedo reticular que o paciente apresentava. Os níveis de metahemoglobina foram respectivamente 29,05 antes do tratamento e 5,2 após a administração do azul do metileno. A criança evolui bem tendo alta da UTI bem em poucos dias após o evento.

\section{DISCUSSÃO}

\section{Óxido nítrico}

Em 1980 Furchgott e Zawadzki ${ }^{1}$ descreveram um fator humoral produzido pelo endotélio mediador da ação da acetilcolina na vasodilatação que posteriormente foi descoberto se tratar do próprio oxido nítrico (NO). Até meados dos anos 1970 as propriedades do gás eram pouco conhecidas e acreditava-se que a molécula não passava de um poluente produzido por reações bacterianas e pela digestão dos humanos. A partir de então diversas pesquisas sobre os efeitos biológicos do NO foram feitas e o seu potencial terapêutico passou a ser utilizado na prática clínica já que, por ser altamente solúvel e possuir ampla distribuição, exerce ação parácrina em variados tecidos.

\section{Mecanismo de ação}

Descrita inicialmente por Rapoport e Murad como "endothelial-derivated relaxing factor" (EDRF), o NO apresenta efeito de dilatação vascular mediado pela sua ligação com o grupo heme da guanilato ciclase (GC) e consequente ativação da enzima, o que proporciona o acúmulo de guanosina monofosfato cíclica (GMPc). Seu mecanismo de relaxamento de musculatura lisa endotelial envolve a inibição de entrada de cálcio na célula (com sequestro do íon pelo retículo endoplasmático) e abertura dos canais membranares de potássio, seguida por hiperpolarização celular e desfosforilação das cadeias leves de miosina.

A partir do achado frequentemente de nitrato em crianças acometidas por diarreias de diversas etiologias, o gás, que é sintetizado in vivo a partir do precursor L-arginina $^{2}$ com a presença da enzima óxido nítrico sintase (NOS), passou a ser considerado um importante associado do sistema imune. Hoje se sabe que é largamente produzido em macrófagos como agente citotóxico e inibe a agregação de neutrófilos.

A consagração do NO endógeno e exógeno como vasodilatador forneceu substrato científico para uma pesquisa realizada ${ }^{3,4}$ uma década antes, que buscava embasar a conduta comum na medicina oriental chinesa de fornecer nitratos orgânicos e nitroglicerina para o tratamento da angina pectoris, insuficiência cardíaca congestiva (ICC) e hipertensão pulmonar (HP). 


\section{Indicações da utilização de NO}

Atualmente o NO vem sendo utilizado como um rápido indutor de vasodilatação que reduz a pressão da artéria pulmonar e aumenta os níveis de oxigenação sanguínea ${ }^{5}$. As principais indicações da utilização de NO são:

Síndrome do desconforto respiratório agudo (SDRS): devido à alteração da produção de surfactante e processo inflamatório, há uma quebra da barreira aoveolocapilar gerando edema intersticial (não homogêneo) e aoveolar e terminando hipoxemia refratária à administração de oxigênio e hipertensão pulmonar com sobrecarga do ventrículo direito;

Anemia Falciforme: benéfico no tratamento das crises vaso oclusivas agudas;

Broncoespasmo: devido ao provável efeito broncodilatador;

Cirrugia cardiaca ${ }^{6}$ : Pode ser utilizado no intraoperatório de cirurgia cardíaca adulta ou pediátrica (cardiopatia congênita) e no transplante cardíaco. A sua utilização visa preservar a perfusão coronariana, otimizar a pré-carga e reduzir a pós-carga do ventrículo direito evitando sua falência, além de não ter ação na pressão arterial sistêmica. Pode ser utilizado também para tratar a HP que pode ocorrer no perioperatório destes pacientes;

Doença pulmonar obstrutiva crônica e fibrose pulmonar: eficaz na redução da hipertensão pulmonar causada pela fibrose pulmonar e remodelação das paredes das artérias pulmonares, além da hipóxia;

Hipertensão pulmonar primária em jovens ${ }^{7}$ : atua na grave hipertrofia da camada íntima da artéria pulmonar.

Recentemente estudos não controlados sobre a aplicação de NO nos casos de hipertensão pulmonar persistente (HPP) do recém-nascido (RN) demonstraram a melhora da oxigenação ${ }^{8}$ embora outros, randomizados e controlados com placebo, não demonstrem queda na mortalidade.

\section{Complicações}

Como complicações, temos a possível formação de resíduos citotóxicos de nitrotirosinas, que promovem a redução da produção de surfactantes ${ }^{9}$. Para que isso seja evitado, o NO deve ser administrado com uma quantidade mínima de $\mathrm{O}$, para evitar a formação de dióxido de nitrogênio $\mathrm{NO}_{2}$ e, consequentemente, dos resíduos de nitrotirosinas. Pode também ocorrer elevação do fluxo sanguíneo pulmonar e consequente aumento da précarga ou hipertensão pulmonar rebote. Outra possível complicação é a formação de metahemoglobina, a MetHb, que será discutida adiante.

\section{Hemoglobina}

A hemoglobina humana é composta por uma estrutura quaternária de cadeias polipeptídicas (globinas) as quais possuem um grupo prostético (heme) em seu interior. Este grupo consiste basicamente em um anel de protoporfitina $\mathrm{IX}^{10}$ complexado a um átomo de ferro em seu estado ferroso $\left(\mathrm{Fe}^{2+}\right)$. Dessa forma, cada molécula de hemoglobina carrega quatro átomos de ferro, cada qual tem a capacidade de ligar-se reversivelmente a uma molécula de $\mathrm{O}_{2}$. A forma de $\mathrm{Hb}$ mais comumente encontrada em adultos é a $\mathrm{HbA}$, cujas cadeias denominam-se alfa 1 , alfa 2 , beta 1 e beta 2 . Podem ocorrer, porém, variantes incapazes de transportar oxigênio adequadamente aos tecidos, chamadas disemoglobinas (disHb), como no caso da carboxihemoglobina $(\mathrm{COHb})$, sulfemohemoglobina $(\mathrm{SHb})$ e da metahemoglobina (MetHb).

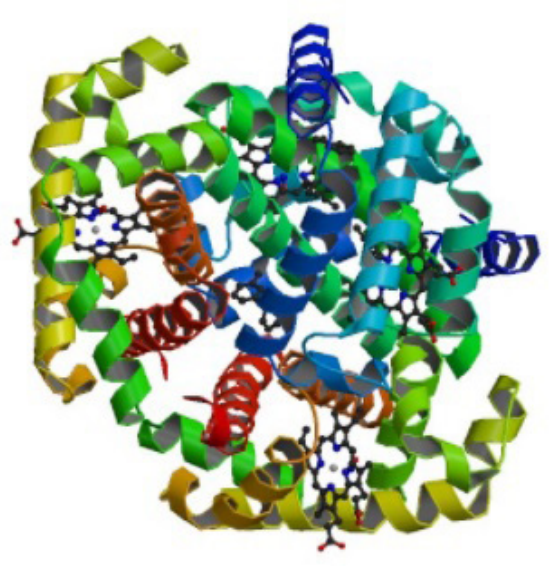

Figura 1. Hemoglobina humana conduzindo NO (Fonte: Available from: http://www.rcsb.org/pdb/explore/explore. do? structureId $=4 \mathrm{~N} 8 \mathrm{~T}$ )

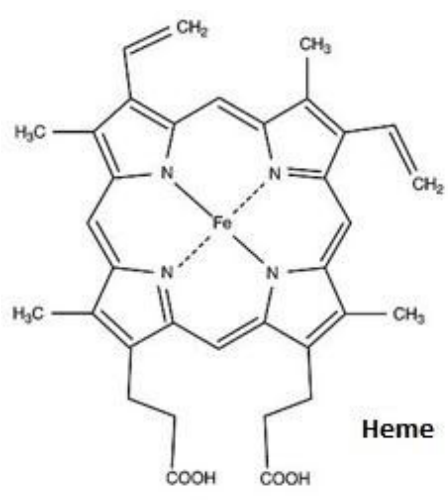

Figura 2. Estrutura primária de subunidade de hemoglobina humana (Fonte: Wikipédia)

\section{Metahemoglobina}

A metahemoglobina (MetHb) é caracterizada por possuir o átomo de ferro da porção heme oxidado ao estado férrico $\left(\mathrm{Fe}^{3+}\right)$, o que o impede que se ligue ao oxigênio. $\mathrm{O}$ estado oxidado do ferro também promove alteração alostérica na porção heme da $\mathrm{Hb}$ parcialmente 
oxidada, e aumenta sua afinidade por $\mathrm{O}_{2}$. Com isso, ocorre desvio da curva de dissociação da $\mathrm{Hb}^{2}$ o que prejudica sua liberação para os tecidos. Dessa forma, a hipóxia tecidual provocada pela MetHb é consequência não só da diminuição da $\mathrm{Hb}$ livre para transportar $\mathrm{O}_{2}$, mas também pela dificuldade de liberação de $\mathrm{O}$ para os tecidos. Uma vez que estejam desequilibrados os sistemas naturais de oxirredução do organismo (sem aumento de produção de MetHb ou redução da atividade enzimática), pode-se instalar a metahemoglobinemia.

\section{Mecanismos de proteção enzimática ${ }^{11}$}

A $\mathrm{Hb}$ é naturalmente oxidada a MetHb pelos processos fisiológicos, mas mecanismos de proteção enzimática são responsáveis sua redução dentro de 3 a 5 minutos e pela manutenção de seus níveis abaixo de $2 \%$.

Os dois mecanismos principais de redução da metahemoglobina são a diaforese I (ou citocromo 5) e diaforese II, ou redutase de hemoglobina dependente de NADH e redutase de metahemoglobina dependente de NADPH respectivamente. Destacam-se também o ácido ascórbico e glutation.

Nos pacientes que têm deficiência de glicose 6 fosfo diesterase (G6PD), a diaforese II não é funcionante, já que não há produção de NADPH, sendo estes mais suscetíveis à metahemoglobinemia.

\section{Deficiência de glicose 6 fosfato desidrogenase}

Patologia hereditária ligada ao gene $\mathrm{X}$, acomete predominantemente e com fenótipo mais grave os homens. Sua epidemiologia não poupa continentes, refletindo seleção ambiental e migração. Encontradas fortes correlações com a epidemiologia da malária: Em 1956,

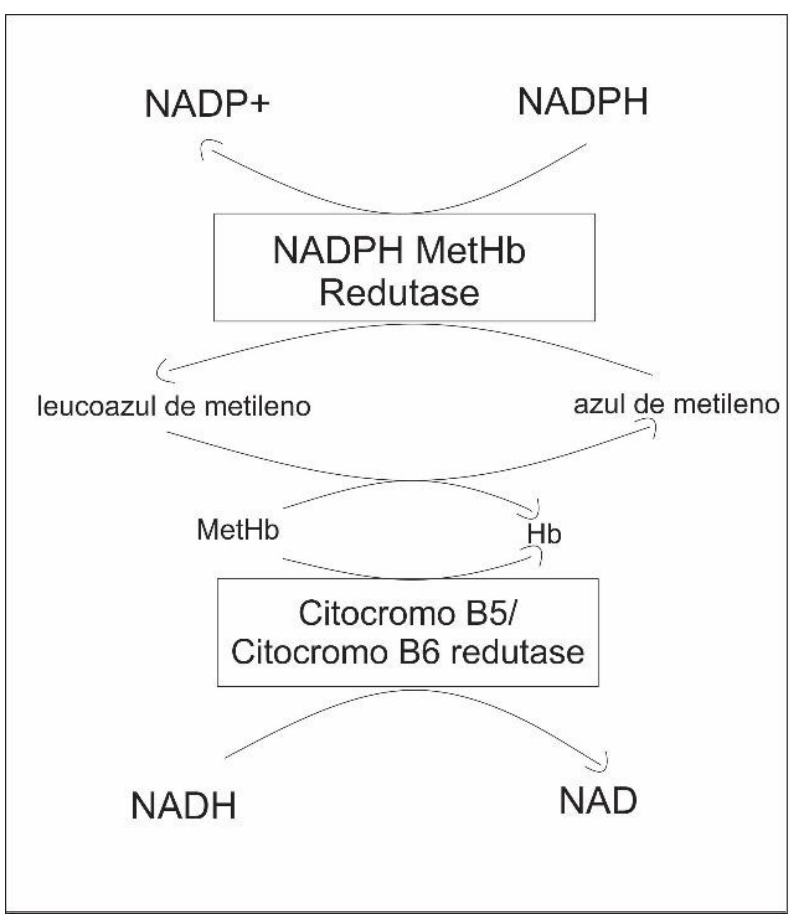

Figura 3. Principal via de redução da metahemoglobina (MetHb) (Modificado a partir de Nascimento et al. ${ }^{10}$, p.652)

um grupo de Chicago relatou que pessoas cujas células vermelhas apresentavam hemólise quando expostas a antimaláricos ${ }^{12}$ eram deficientes em G6PD (actividade enzimática $<15 \%$ do normal), e em 1958 uma equipe italiana comprovou a mesma deficiência em crianças com uma história prévia de favismo.

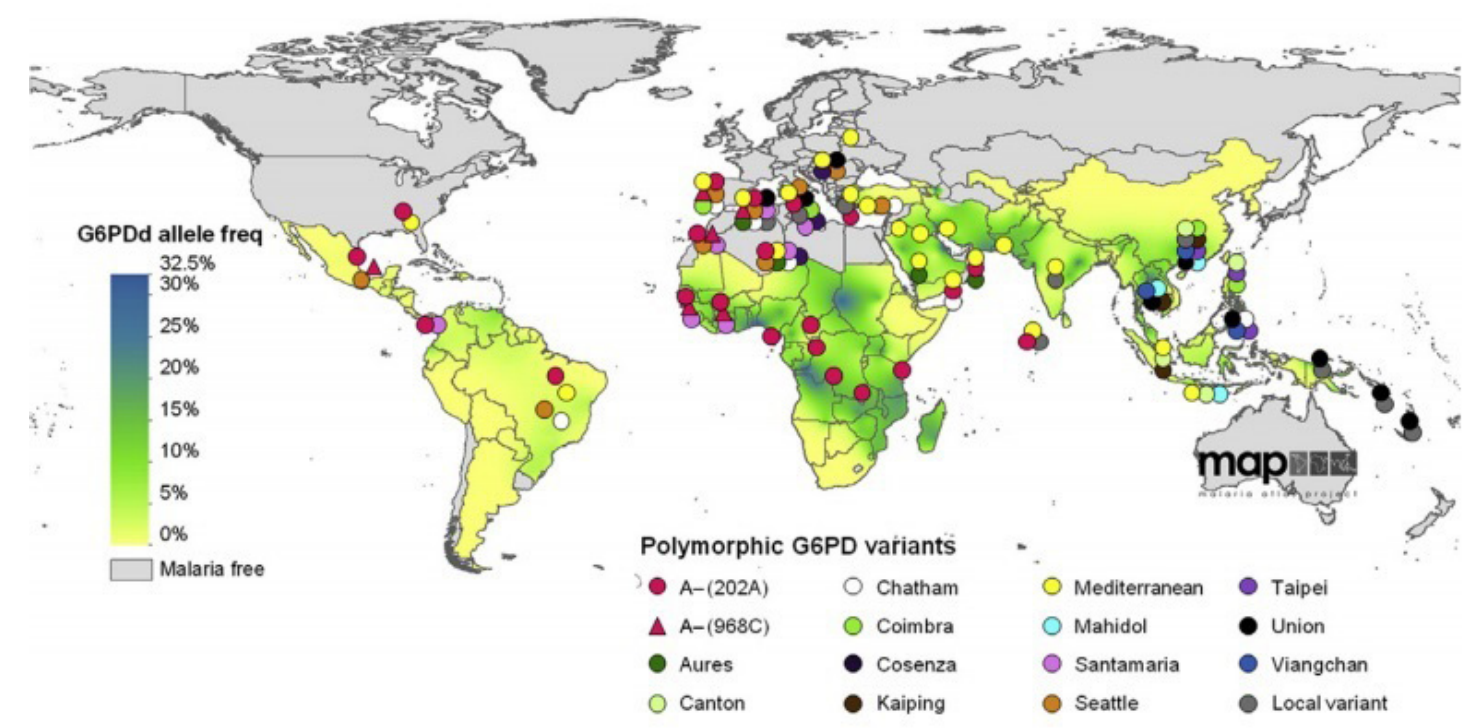

Fonte: Luzzatto et al. ${ }^{10}$, p.380.

Figura 4. Distribuição da Deficiência de G6PD no mundo 


\section{Características clínicas da deficiência de glicose 6 fosfato desidrogenase}

Os pacientes apresentam predisposição à hemólise (com quedas dos níveis de hemoglobina dos valores normais a até $4 \mathrm{mg} / \mathrm{dl}$ em $48 \mathrm{~h}$ ), sendo as manifestações mais comuns icterícia neonatal que se não tratada pode levar a danos neurológicos, anemia hemolítica aguda e hemoglobinúria macroscópica. Em adultos, no entanto, a patologia pode permanecer assintomática por longos períodos. Os principais fatores desencadeantes dos sintomas são ingestão de fava, inalação de pólen e alguns fármacos como primaquina (antimalárico), azul de metileno, e alguns medicamentos utilizados para afecções do trato urinário (pyridium, nitrofurantoína).

\section{Metahemoglobinemia}

Níveis acima de $2 \%$ de MetHb podem estar associados a patologias congênitas e, acredita-se que mais frequentemente, à exposição a agentes oxidantes diversos. Em crianças, pode ocorrer metahemoglobinemia em decorrência de sepse, crise falcêmica e alterações gastrointestinais, uma vez que nestes indivíduos as concentrações das enzimas redutoras de $\mathrm{Hb}$ são em média $50 \%$ menores do que as esperadas para um adulto.

\section{Causas congênitas de metahemoglobinemia}

Mutações no gene $\mathrm{CYB}^{2} 3^{13}$, podem induzir a deficiência da enzima citocromo 5 redutase levando à metahemoglobinemia congênita tipo I, restrita aos eritrócitos e tipo II, caracterizada por acometimento sistêmico.

\section{Causas adquiridas de metahemoglobinemia}

As formas adquiridas (agudas) ocorrem devido à sobreposição dos fatores oxidantes externos ao poder redutor endógeno. Nesse sentido, fertilizantes, pesticidas, herbicidas, fumaça automobilística, anilina industrial e diversos tipos de alimentos podem se relacionar ao desenvolvimento da metahemoglobinemia.

Dentre os fármacos destacam-se os anestésicos locais (benzocaína, lindocaína, prilocaína ${ }^{14}$ ) rotineiramente utilizados em consultórios odontológicos, a dapsona (utilizada no tratamento de hanseníase), derivados de fenacetina e antimaláricos. Vale ressaltar que o risco de elevação dos níveis sanguíneo de MetHb é maior quando a administração de tais fármacos se dá diretamente em mucosas, o que levou a agência reguladora dos EUA, a Foods and Drugs Administration (FDA) à proibição do emprego de sprays de benzocaína em região orofaríngea durante procedimentos de endoscopia.

As intoxicações resultantes em MetHb geralmente têm como responsáveis os nitritos e nitratos (cuja absorção é facilitada por bactérias do trato gastrointestinal) , presentes em beterrabas, cenoura e espinafre mas em maior grau em alimentos industrializados, devido a seu importante papel adjuvante em conservantes e corantes.

Lactentes são especialmente sensíveis a desenvolver metahemoglobinemia devido à presença de hemoglobina fetal $\mathrm{HbF}$ (mais facilmente oxidada que $\mathrm{HbA}$ ), além de menor atuação da enzima CB5R (50 a 60\% inferior do que nos adultos até os 4 meses). Ainda, o pH intestinal dessa população facilita o crescimento de bactérias Gram, responsáveis pela conversão de nitratos em nitritos.

Quadro 1. Modificado a partir de Nascimento et al. ${ }^{10}$

\begin{tabular}{|l|l|}
\hline \multicolumn{2}{|c|}{ Fármacos capazes de induzir metahemoglobinemia } \\
\hline Acetaminofeno & Dapsona \\
Ácido p-salicilico & Hipoglicemiantes orais \\
Benzocaína & Nitrato de amônio \\
Bupivacaína & Nitrato de prata \\
Lindocaína & Nitrato de sódio \\
Prilocaína & Nitroglicerinas \\
Anticonvulsivantes & Óxido nítrico \\
Ácido valpróico & Riluzole \\
Fenitpína & Sulfonamidas \\
Antimaláricos & Sulfametotaxol \\
Cloroquina & Sulfatiazidas \\
\hline
\end{tabular}

\section{Quadro clínico}

Diante da suspeita de metahemoglobulinemia, considerar se o quadro é agudo ou crônico e a intensidade dos sintomas. Geralmente os sintomas são vagos e inespecíficos que aumentam proporcionalmente a elevação dos níveis de metahemoglobinemia. Valores entre $10 \mathrm{e}$ $20 \%$ levam à cianose, entre 20 a 50\% estresse respiratório, tontura, cefaleia e fadiga. Ao atingir 50 a $70 \%$ o paciente pode apresentar letargia e torpor. Pacientes portadores de comorbidades que prejudicam a oxigenação sanguínea (como cardiopatias, DPOC, pneumonia e anemia) podem apresentar sintomas com menores níveis de metahemoglobinemia.

Aferições ambulatoriais de saturação como o oxímetro de pulso e gasometria arterial não excluem o diagnóstico de metahemoglobinemia, uma vez que em presença de hemoglobina anormal o oxigênio continua sendo entregue dissolvido pelos pulmões, apesar de o gás não estar realmente disponível para utilização celular, o que poderia gerar uma falsa ideia de oxigenação.

Todos os pacientes cianóticos não responsivos a oxigenioterapia e com $\mathrm{PaO}_{2}$ normal e sangue arterial de coloração acastanhada devem ser investigados para metahemoglobinemia. 
Quadro 2. Modificado a partir deNascimento et al. ${ }^{10}$

\begin{tabular}{|l|l|}
\hline \multicolumn{2}{|c|}{ Manifestações clínicas da metahemoglobinemia } \\
\hline MetHb (\%) & \multicolumn{1}{c|}{ Sinais e sintomas } \\
\hline$<3$ (normal) & nenhum \\
\hline 3 a 15 & Pele acinzentada ou ausentes \\
\hline 15 a 30 & Cianose, sangue acastanhado \\
\hline 30 a 50 & Dispnéia, cefaléia, fraqueza, vertigem \\
\hline 50 a 70 & $\begin{array}{l}\text { Taquidispnéia, acidose metabólica, } \\
\text { arritmias cardíacas, convulsões, coma }\end{array}$ \\
\hline$>70$ & Torpor, óbito \\
\hline
\end{tabular}

\section{REFERÊNCIAS}

1. Dusse LMS, Vieira LM, Carvalho MG. Revisão sobre óxido nítrico. J Bras Patol Med Lab. 2003;39(4):343-50. Disponível em: http://www.scielo.br/pdf/jbpml/v39n4/18548.pdf.

2. Pavan B, Crawford J, Hall M, Lang JD Jr. Inhaled nitric oxide: current clinical concepts. Nitric Oxide. 2015;50:114-28. doi: 10.1016/j.niox.2015.08.007.

3. Katsuki SA, Arnold W, Mittal C, Murad F. Stimulation of guanylate cyclase by sodium nitroprusside, nitroglycerin and nitric oxide in various tissue preparations and comparison to the effects of sodium azide and hydroxylamine. J Cyclic Nucleotide Res. 1977;3:23-5.

4. Schultz KD, Schultz K, Schultz G. Sodium nitroprussiate and other smooth muscle relaxants increase cyclic GMP levels in rat ductus deferent. Nature. 1977;265:750-1.

5. Salguero KL, Cummings JJ. Inhaled nitric oxide and methemoglobin in full-term infants with persistent pulmonary hypertension of the newborn. Pulmonary Pharmacol Ther. 2002;15(1):1-5. doi: 10.1006/pupt.2001.0311.

6. Santini F, Casali G, Franchi G, Auriemma S, Lusini M, Barozzi L, Favaro A, Messina A, Mazzucco A. Hemodynamic effects of inhaled nitric oxide and phosphodiesterase inhibitor (dipyridamole) on secondary pulmonary hypertension following heart valve surgery in adults. Int J Cardiol. 2005;103(2):156-63. doi: 10.1016/j.ijcard.2004.08.050.

7. George I, Xydas S, Topkara VK, Ferdinando C, Barnwell EC, Gableman L, Sladen RN, Naka Y, Mehmet C. Oz. Clinical indication for use and outcomes after inhaled nitric oxide therapy. Ann Thorac Surg. 2006;82(6):2161-9. doi: 10.1016/j.
O manejo clínico da metahemoglobinemia depende do reconhecimento dos sintomas como cianose central, valores de oximetria de pulso que não respondem a oxigenoterapia e alteração do estado de consciência. A droga de escolha, nem sempre eficaz é o azul de metileno, utilizada na dose de 1 a $3 \mathrm{mg} / \mathrm{kg}$ de peso corporal endovenoso (EV) em 5 minutos além da suspensão imediata do fator desencadeante.

\section{CONCLUSÃO}

Metahemoglobinemia pode ser suspeitada em pacientes que utilizam NO. Níveis elevados de metahemoglobina no sangue podem ser incompatíveis com a vida. O tratamento deve ser instituído de forma rápida e eficaz.

athoracsur.2006.06.081.

8. Fioretto JR. Uso do óxido nítrico em pediatria. J Pediatr (Rio J.). 2003;79(Suppl 2):S177-S186. Disponível em: http:// www.scielo.br/pdf/jped/v79s2/v79s2a07.pdf.

9. Gurgueira GL, Carvalho WB. Óxido nítrico inalatório: considerações sobre sua aplicação clínica. J Pneumol. 2003;29(5):325-31. http://dx.doi.org/10.1590/S010235862003000500012 .

10. Nascimento TS, Pereira ROL, Mello HLD, Costa J. Metahemoglobinemia: del diagnóstico al tratamiento. Rev Bras Anestesiol. 2008;58(6):651-64. http://dx.doi. org/10.1590/S0034-70942008000600011.

11. Rodrigues DF, Vieira FC, Rodrigues MEF, Metahemoglobinemia: etiopatogenia e quadro clínico. Rev Pediatria SOPERJ. 2011;12 (1):8-11. Disponível em: http:// revistadepediatriasoperj.org.br/detalhe_artigo.asp?id=563.

12. Luzzatto L, Nannelli C, Notaro R. Glucose-6-phosphate dehydrogenase deficiency. Hematol Oncol Clin North Am. 2016;30(2):373-93. doi: 10.1016/j.hoc.2015.11.006.

13. Cooper MS, Randall M, Rowell M, Charlton M, Greenway A, Barnes C. Congenital methemoglobinemia type II - clinical improvement with short-term methylene blue Treatment. Pediatr Blood Cancer. 2016;63:558-60. doi:10.1002/ pbc.25791.

14. Trapp L, Will J. Acquired methemoglobinemia. Revisited, Dental Clinics of North America. 2010;54(4): 665-75. doi: 10.1016/j.cden.2010.06.007. 